\title{
LEADER-DRIVEN MULTICAST FOR VIDEO STREAMING ON WIRELESS LANS
}

\author{
PENG GE AND PHILIP K. MCKINLEY \\ Software Engineering and Network Systems Laboratory \\ Department of Computer Science and Engineering \\ Michigan State University \\ East Lansing, Michigan 48824 \\ E-mail: \{gepeng,mckinley\}@cse.msu.edu
}

\begin{abstract}
The IEEE 802.11 MAC layer uses RTS/CTS/ACK signaling to improve the reliability of unicast transmissions, but in current products there is no special signaling support for multicast transmissions. Therefore, the packet loss rate of multicast communication is generally higher than that of unicast communication. This paper evaluates a new MAC-layer multicasting technique called Leader-Driven Multicast $(L D M)$. Instead of multicasting to a group address, the source node sends a unicast stream to the address of a leader node among the receivers. All other receivers passively receive this unicast stream, including retransmissions. This paper investigates the effects of the LDM protocol on interactive video multicasting across WLANs. Results are presented from a simulation study using different packet loss models and different loss correlations among receivers. The results indicate that LDM is potentially beneficial even when there is no loss correlation among receivers. When losses do exhibit some positive correlation LDM can provide a very high quality multicast stream to WLAN nodes, especially when combined with application-level error control techniques.
\end{abstract}

\section{Introduction}

Rapid advances in wireless packet networking technology are making it possible to provide mobile users with not only voice and data connections, but also with video communication services. Many emerging mobile applications involve the delivery of video streams to mobile hosts (MHs). In particular, wireless local area networks (WLANs) provide sufficient bandwidth to support compressed video transmission, although steps must be taken to reduce packet loss due to noise and interference. One situation that presents a unique set of problems is video multicast, in which multiple MHs want to receive the same video stream from a single source. Sending separate unicast streams to multiple receivers is prohibitive for large groups. A more efficient approach is to use multicast services provided by IP and MAC layer protocols.

Current WLAN technologies do not support multicast well, however. For unicast transmissions, WLANs based on the IEEE 802.11 standard use RTS/CTS signaling and link-level acknowledgements to reduce collisions and

main: submitted to World Scientific on June 6, 2002 
enhance reliability. On the other hand, multicast frames are transmitted without either of these mechanisms, and hence multicast transmissions suffer higher loss rates than their unicast counterparts [1]. In response to this problem, a number of proposals have been put forward to enhance the reliability and the efficiency of multicast in the 802.11 protocol suite [2-4]. Most of these protocols involve feedback (CTS and/or NACK signals) from multiple receivers. However, our experiments show that collisions involving reverse traffic can be a major impediment to performance in WLANs, and that even global suppression techniques are ineffective for large groups of receivers [5].

To improve multicast performance while minimizing feedback traffic, we recently proposed a WLAN MAC-layer enhancement called leader-driven multicast (LDM) [6]. Instead of transmitting a multicast stream to a group address, the source node sends a unicast stream to a leader node among the receivers. All other receivers passively receive the unicast stream, including retransmissions. The quality of the stream received by the leader is very high, due to unicast RTS/CTS signaling and MAC-level retransmissions by the sending node. Moreover, our results show that these retransmissions can also produce a residual effect and improve reception at other receivers.

In this paper, we evaluate the LDM protocol as used to support interactive video transmission across WLANs. Sample results are presented from a simulation study in which we used three different packet loss models and varied the loss correlation among receivers. The results indicate that LDM is potentially beneficial even when there is no loss correlation among receivers. When losses do exhibit some correlation (which we have observed in our own testbed), LDM can be very helpful, especially when combined with application-level error control methods.

\section{Error Control Methods}

Our investigations consider three approaches to video error control. Each is briefly described below.

\subsection{Leader-Driven Multicast (LDM)}

The first error control method, LDM, requires a small modification to the IEEE 802.11 MAC layer protocol. As described above, the sender (often the access point in the distribution of video to a group of wireless hosts) transmits a unicast stream to a leader $\mathrm{MH}$, and other $\mathrm{MHs}$ in the session monitor this unicast channel. Clearly, LDM will improve the reception rate of the leader, compared to pure multicast, due to the unicast signaling and

main: submitted to World Scientific on June 6, 2002 
acknowledgements at the MAC layer. Moreover, the reception rate at other receivers may also improve, if the MAC layer retransmissions for the leader happen to cover some of the packet losses of those nodes.

Furthermore, LDM eliminates the need for pure multicast in the forward traffic stream. The advantage of this change can be seen in the following example. Assume that two concurrent multicast sessions are using the same WLAN. When using pure multicast, each session has no knowledge of the other session, and therefore uses the channel at its own convenience. In the worst case, this behavior will cause the collision of many data packets for both sessions. On the other hand, by using LDM, each session has to reserve the wireless channel with RTS/CTS signaling before it can transmit a data packet, which should produce more controlled channel access and, hence, fewer collisions.

A potential drawback of LDM is that a leader with poor reception might lead to higher bandwidth consumption than in pure multicast, due to excessive data retransmissions. In addition, allowing a MH to monitor unicast traffic raises a security issue. However, we believe that this issue can be handled through the use of existing encryption algorithms and public/private key management protocols. Moreover, the same problem is present in wired LANs, where any node connected to the network can access all the data packets.

\subsection{Forward Error Correction for Video.}

The second error control method is to apply forward error correction(FEC) to the video stream. Block erasure codes such as those implemented by Rizzo [7] are parameterized by $n$ and $k$. For every $k$ data packets to be transmitted, the code is invoked to produce $n-k$ parity packets. As the protocol at the sender accumulates the data packets for a given group, it also forwards those data packets to the receivers. In proactive FEC, some or all of the the parity packets are transmitted along with the data packets. In reactive FEC, some or all of the parity packets (instead of data packets) are transmitted in response to NACKs. If at least $k$ of the $n$ packets in a given group arrive at a receiver, then the $k$ data packets can be reconstructed using FEC decoding. These codes are ideal for multicasting, since a single parity packet can correct different losses at multiple receivers [8].

One problem in applying block erasure codes to video streaming is that many video frames are small and do not require many packets. Allowing an FEC group to span two (or more) frames would cause undue delay in delivery and in decoding of the first frame, and would risk missing playback deadlines. To solve this problem, we use dummy packets [9]. In this approach, we invoke

main: submitted to World Scientific on June 6, 2002 
the FEC code using the data packets in the frame, plus an appropriate number of packets that are initialized to all 0's. These dummy packets are used only for computing the parity packets, and are not actually sent out into the channel. The application level header of each packet contains information on the FEC parameters, frame size, and the number of dummy packets, enabling the receiver to decode the packets correctly.

In addition to dummy packets, we also make use of QoS-directed error control (QDEC), a technique proposed by Xu et al. [10] for video multicast in wireless networks. Those authors argued that compressed video formats, such as MPEG, usually involve encoded frames of varying importance, and therefore, error control should be performed in a differentiated manner. In the case of an MPEG-1 video, for example, QDEC applies FEC to I frames and P frames, while providing no redundancy for B frames. Since I and P frames are more important than B frames in the MPEG stream, this approach provides a good tradeoff between QoS and the bandwidth consumed in WLANs. Specific QDEC parameter settings are discussed later.

\subsection{Extra Parity Request (EPR)}

The third method, EPR, involves feedback from selected receivers to a proxy node in the wired network. For each FEC group, the proxy computes $n-k$ parity packets. In order to save the bandwidth, however, the proxy initially sends only a subset of these parity packets, with additional parity packets sent upon request from responders (MHs that may request extra parity packets) that receive fewer than $k$ packets in the group. In the case of video streams, whether or not a responder should request extra parity packets depends on whether those additional packets can be received without missing the playback deadline of that frame. For first generation 2Mbps WLANs, meeting this deadline is difficult, at least for large frames. On an $11 \mathrm{Mbps}$ network with a 6Mbps effective bandwidth, however, a typical MPEG-1 I frame requires only $25 \mathrm{~ms}$ to be transmitted. By inserting a relatively small delay $(100 \mathrm{~ms}$ or so) in playing back the video stream at the $\mathrm{MH}$, the overhead for reactive error control can easily be absorbed without significantly affecting the video quality. We refer to this technique as Extra Parity Request (EPR). In our prior work [1], we implemented a proxy server that supports both QDEC and EPR, and showed that the combination can dramatically improve the frame delivery rate of video streams. In our current project, we evaluate the effects of an LDM-capable MAC layer on performance, when combined with QDEC and/or EPR.

If EPR is enabled, then the issue of NAK suppression arises. Specifically,

main: submitted to World Scientific on June 6, 2002 
as the number of MHs increases, the protocol risks NAK implosion at the proxy. To address this problem, we use global NAK suppression [11]. Instead of unicasting NAKs to the proxy alone, the receiver multicasts the NAK, so that it is heard by all $\mathrm{MHs}$ within range. A simple timer at each $\mathrm{MH}$ is used to introduce a small random delay for each NAK. If another MH hears the NAK and determines that its own pending NAK is subsumed, then it cancels its NAK. Similarly, for a given FEC group and a given time window, the proxy responds only to the NAK requesting the most packets.

\section{Simulation Environment}

We conducted our simulation study of video error control using MX [12], a simulation tool that enables unmodified application programs to be executed atop a simulated network. In MX, a file written in a configuration description language describes the characteristics of the network components: host computers, routers, operating system delays, network interface card properties, and so on. A socket-level API enables application code (either $\mathrm{C}++$ or Java) to be linked with the simulator. Protocol modules in a virtual node are implemented as objects with uniform interfaces; multiple modules can be linked together to form a protocol stack. Supported protocol modules include TCP, UDP, IP, CSMA/CD, MACA, and IEEE 802.11, and this set can be easily expanded. Both correlated and uncorrelated error behavior can be modeled on physical links, using either analytical distributions or trace-based inputs.

We used MX to model an environment similar to our own testbed, except for the numbers of MHs. Figure 1 shows the physical configuration. The sender and proxy are executed on dual $450 \mathrm{MHz}$ workstations connected by $100 \mathrm{Mbps}$ Ethernet. The MHs are $300 \mathrm{MHz}$ laptops connected by an $11 \mathrm{Mbps}$ Cisco Aironet WLAN. The number of receivers is varied from 1 to 25. To simulate LDM, we modified the 802.11 b protocol module to enable each $\mathrm{MH}$ to monitor packets destined for specified unicast addresses (effectively, a "targeted" RF monitoring mode).

The video stream we used for the simulation is the same one that we used in earlier experiments [1]. This MPEG-1 stream has 412 frames in 13.7 seconds (we are currently conducting a follow-on study that addresses proxybased error control for MPEG-4 streams). MPEG-1 includes three types of frames: I frames coded as still images, $\mathrm{P}$ frames predicted from the most recent I or P frame, and B frames predicted from the closest two surrounding I or $\mathrm{P}$ frames. The typical 12-frame pattern, and the one used in our tests, is IBBPBBPBBPBB. While other video encoding methods such as H.263 and MPEG-4 provide lower bit rates, our goal in this study was to investigate

main: submitted to World Scientific on June 6, 2002 


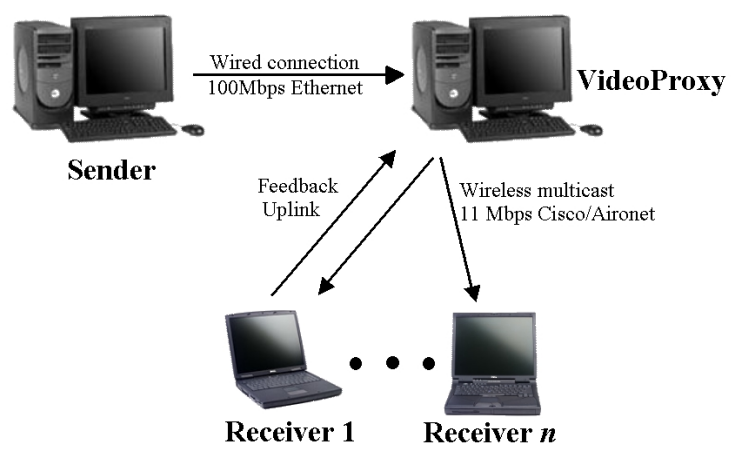

Figure 1. Experimental configuration modeled in simulations.

the issues in delivering full MPEG-1 streams across wireless networks. The MPEG file size is 1.33 Mbytes, which produces an average bandwidth consumed around $800 \mathrm{Kbps}$. In our test data, I and $\mathrm{P}$ frames average approximately 6 Kbytes in size, and B frames 2 Kbytes. Therefore, the raw reception rate is higher for $\mathrm{B}$ frame than for $\mathrm{I}$ and $\mathrm{P}$ frames, using either pure multicast or leader-driven multicast (LDM).

We used three different packet loss models in the simulations. In the simple random model, each packet is lost independently with probability $p$. In the more widely-used two-state Markov model [13], the loss status of each packet depends on the status of the previous packet. The third model, which we refer to as the ParEx model, is based on experimental traces of error burst behavior [5]. In this model, packet burst error lengths are modeled by a Pareto distribution, and error-free burst lengths are modeled by an exponential distribution. For each loss model, we vary parameters to generate different packet loss rates.

We also vary the packet loss correlation among different receivers. Although many studies on WLAN packet loss behavior assume that losses are totally independent, results of traces in our laboratory show that correlation can actually be quite high. Based on traces of losses at MHs, we calculated the loss covariance of each trace pair, using the same approach as in [14] to calculate covariance. Then we compute the correlation coefficients. Even when queueing losses at the access point are excluded from consideration, the loss correlation was found sometimes to be as high as 30\% [5]. Of course, LDM is most effective the correlation in packet losses between the leader and

main: submitted to World Scientific on June 6, 2002 
the other MHs is high. However, even when the correlation in packet losses between the leader and any $\mathrm{MH}$ is low, the expected packet delivery rate at non-leader MHs should equal or surpass that of pure multicast transmissions.

\section{Simulation Results}

\subsection{LDM vs $P M$}

In the first group of simulation tests, we transmit only the plain video stream (that is, without FEC), enabling us to assess the effects of LDM versus pure multicast $(\mathrm{PM})$. We vary the loss model of wireless channel as well as the parameters of the models to produce different runs. However, we assume no packet loss correlation among different $\mathrm{MHs}$, which should reveal the minimum improvement of LDM over PM. Figures 2-4, respectively, plot the packet delivery rate and frame delivery rate under each of the three models. In all cases, the reception rate at the LDM leader is high. In these tests, the maximum number of MAC layer transmissios for a frame is set to 4 . Hence, under heavy losses, some of the unicast packets are not delivered even to the leader node. The non-leader receivers in LDM suffer more packet losses than the leader, but they are always better than any receiver using PM. This is the result we expected: LDM can provide significant improvement for the leader, and the reception rate at the other receivers also improves due to the residual effect of MAC-level retransmissions to the leader. The advantage of LDM over PM is more apparent for higher loss rates, because MHs other than the leader are more likely to receive useful retransmissions.

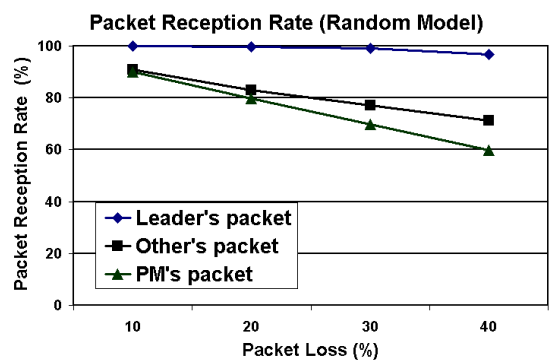

(a) packet reception rate

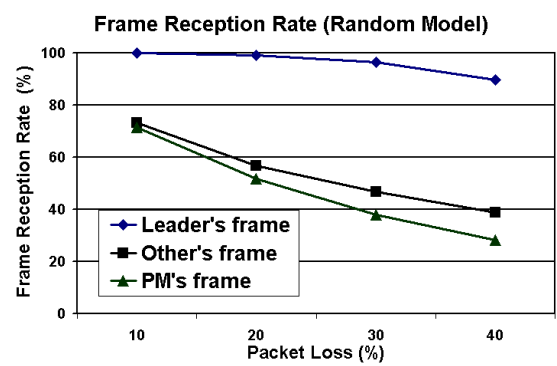

(b) frame reception rate

Figure 2. LDM vs. PM for random loss model. 


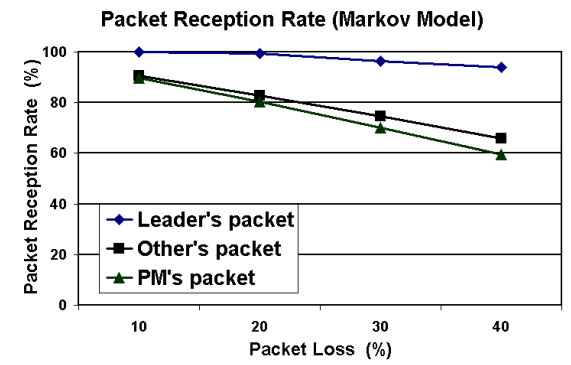

(a) packet reception rate

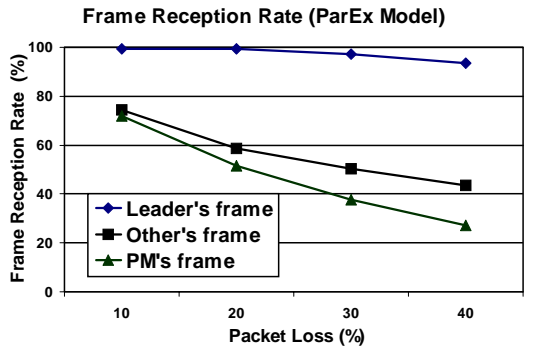

(b) frame reception rate

Figure 3. LDM vs. PM for Markov loss model.

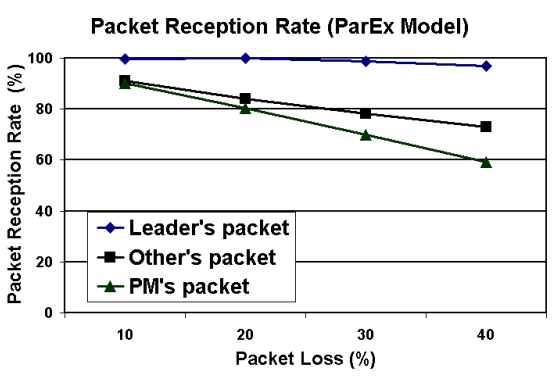

(a) packet reception rate
Frame Reception Rate (ParEx Model)

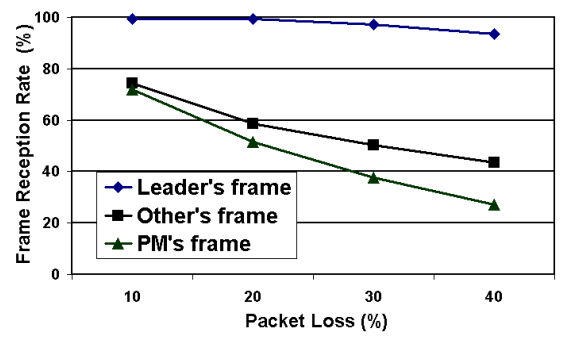

(b) frame reception rate

Figure 4. LDM vs. PM for ParEx loss model.

\subsection{Effect of Packet Loss Correlation}

In the previous set of tests, we assumed that packet losses among different MHs are independent. However, common packet losses among two or more nodes can still occur. Indeed, such random events are the reason for improvement of LDM over PM. In the next set of tests, we used simulation to explore the effects of a positive correlation in packet losses among different MHs. Figure 5 demonstrates the potential improvement in packet reception rate of LDM over PM, for different correlation coefficients, using simulations with both the Markov and ParEx loss models. Four pairs of PM and LDM curves are shown in each plot, for 10\%, 20\%, 30\% and $40 \%$ overall loss rate, respectively. As demonstrated in the plots, when the correlation approaches 
$100 \%$, the reception rate of $\mathrm{LDM}$ also approaches $100 \%$, while the reception rate of $\mathrm{PM}$ is relatively constant.

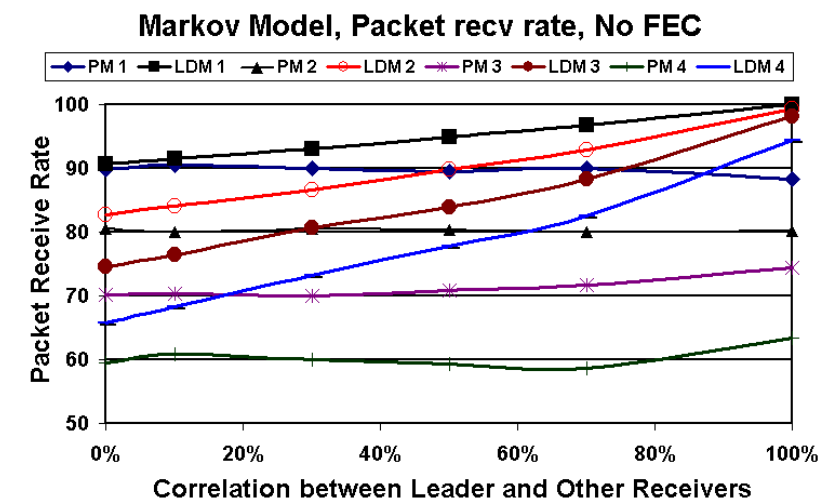

(a) Markov model

ParEx Model, Packet recv rate, No FEC

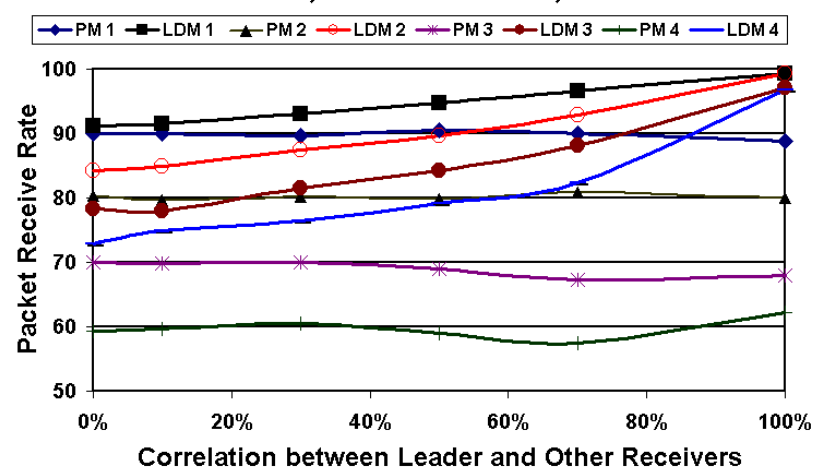

(b) ParEx model

Figure 5. LDM vs. PM with positive packet loss correlation.

\subsection{Combining LDM and FEC}

In the next group of tests discussed here, we apply FEC to the video stream and compare the performance of LDM and PM. The FEC rate is $60-40-20 \%$ for I-P-B frames, respectively. A $60 \%$ FEC rate for I frames means that, if the 
number of data packets for this I frame is $d$, then the number of parity packets sent pro-actively for that group is $\lceil 0.6 d\rceil$. Additional parity packets can be sent when EPR is used. The correlation in packet losses between non-leader $\mathrm{MHs}$ and the leader $\mathrm{MH}$ is set to $30 \%$, and the packet size is 1100 bytes. Figure 6 shows the results for I-frame delivery rate, with and without FEC, for the two loss models and four loss rates.

For all combinations, the delivery rate of the LDM leader is higher than that of other receivers in LDM, which is higher than that of receivers in PM. Again, the advantage is more apparent when the channel quality becomes worse. Of particular note is the very high I frame reception rate for nonleader MHs (over $90 \%$ in all scenarios except one, Markov 40\%, where the rate is $86 \%$ ). Clearly, the combination of LDM and FEC is an effective error control approach under these circumstances.

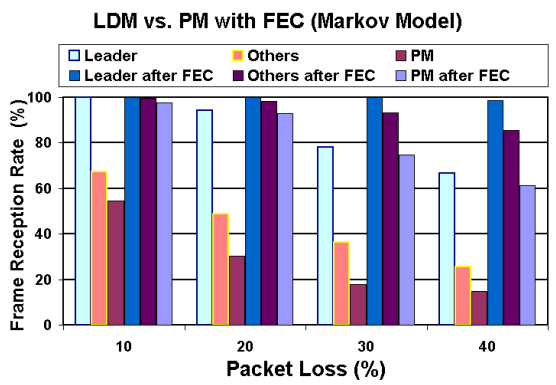

(a) Markov model

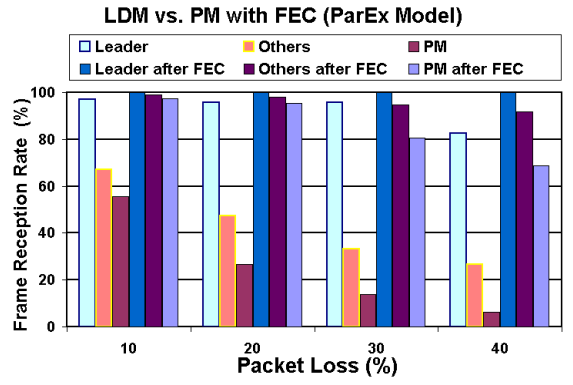

(b) ParEx model

Figure 6. Applying 60-40-20 FEC (only I-frames shown).

\subsection{Combining FEC with EPR}

In the final set of simulation tests, we sought to determine whether or not EPR can improve the delivery of the video stream, and if so, whether or not it is better when combined with LDM or PM. An FEC rate of $60-60-0 \%$ is used for the forward traffic. The number of responders is varied from 1 to 25, Markov model is used. Figure 7 shows the percentage of frames that are delivered successfully.

In most cases, LDM shows better reception than PM. Also, using more responders does not imply better frame delivery, especially when the quality of the wireless channel is bad (e.g., $40 \%$ loss rate). Shown in figure 7 (b), the frame delivery rate achieve maximum when the number of responders is 5 . 


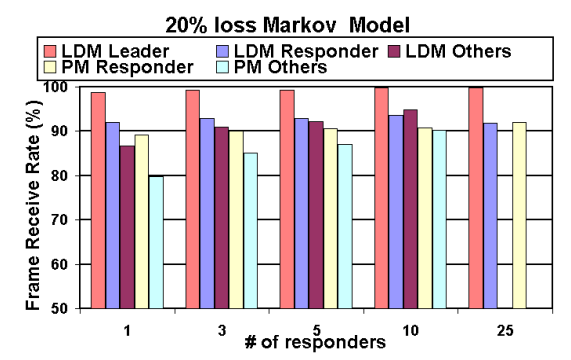

(a) $20 \%$ Loss Markov model

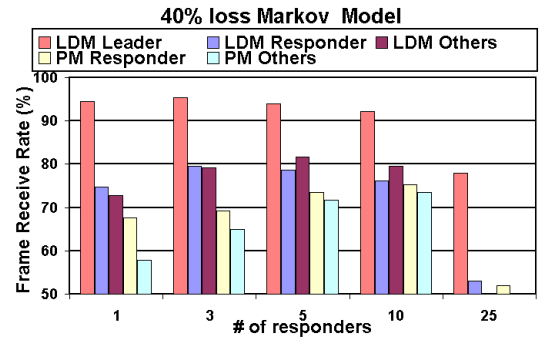

(b) $40 \%$ Loss Markov model

Figure 7. Effect of number of responders.

\section{Conclusions}

In this study, we evaluated the performance of leader-driven multicast, an enhancement to the IEEE 802.11 standard, when applied to video streaming. Simulation results shows that LDM can improve the rate of multicast packet (and frame) delivery in WLANs. The packet/frame reception rate at the LDM leader is very high due to MAC-layer retransmissions, and that at other receivers is higher than when using pure multicast. Moreover, when combined with application-level error control methods, such as forward error correction, the packet and frame delivery rates can be raised to even higher levels, relative to pure multicast.

\section{Acknowledgments}

The authors would like to thank Arun Mani and Chiping Tang for their contributions to this work. This work was supported in part by the U.S. Department of the Navy, Office of Naval Research under Grant No. N00014-01-1-0744, and in part by National Science Foundation grants CDA-9617310, NCR-9706285, CCR-9912407, EIA-0000433, and EIA-0130724.

\section{References}

1. P. Ge and P. K. McKinley, "Experimental evaluation of error control for video multicast over wireless LANs," in Proceedings of the Third International Workshop on Multimedia Network Systems, (Phoenix, Arizona), April 2001.

2. K. Tang and M. Gerla, "MAC layer broadcast support in 802.11 wireless networks," in Proceedings of IEEE MILCOM, 2000, October 2000.

main: submitted to World Scientific on June 6, 2002 
3. K. Tang and M. Gerla, "Random access MAC for efficient broadcast support in ad hoc networks," in Proceedings of IEEE WCNC, 2000, September 2000.

4. J. Kuri and S. Kasera, "Reliable multicast in multi-access wireless LANs," in Proceedings of IEEE Infocom, 1999, March 1999.

5. C. Tang, "Adaptive Reliable Multicast in Wireless Local Area Networks," Master's Thesis, Department of Computer Science, Michigan State University, 2002.

6. P. Ge and P. K. McKinley, "Comparisons of error control techniques for wireless video multicasting," in Proceedings of the IEEE International Performance, Computing, and Communications Conference, (Phoenix, Arizona), April 2002.

7. L. Rizzo, "Effective erasure codes for reliable computer communication protocols," ACM Computer Communication Review, April 1997.

8. L. Rizzo and L. Vicisano, "RMDP: An FEC-based reliable multicast protocol for wireless environments," ACM Mobile Computer and Communication Review, vol. 2, April 1998.

9. P. K. McKinley and A. P. Mani, "An experimental study of adaptive forward error correction for wireless collaborative computing," in Proceedings of the IEEE 2001 Symposium on Applications and the Internet (SAINT-01), (San Diego-Mission Valley, California), January 2001.

10. D. Xu, B. Li, and K. Nahrstedt, "Qos-directed error control of video multicast in wireless networks," in Proceedings of IEEE International Conference on Computer Communications and Networks, October 1999.

11. S. Floyd, V. Jacobson, S. McCanne, C.-G. Liu, and L. Zhang, "A reliable multicast framework for light-weight sessions and applications level framing," in Proceedings of SIGCOMM '95 (Cambridge, MA USA), pp. 342-356, 1995.

12. C. Tang and P. K. McKinley, "MX: A tool for emulation and simulation of distributed applications and protocols," Tech. Rep. MSU-CSE-01-19, Computer Science and Engineering, Michigan State University, East Lansing, Michigan, June 2001.

13. E. N. Gilbert, "Capacity of a burst-noise channel," Bell System Technical Journal, vol. 39, pp. 1253-1265, September 1960.

14. M. Yajnick, J. Kurose, and D.Towsley, "Packet loss correlation in the Mbone multicast network," Tech. Rep. 96-32, Department of Computer Science, University of Massachusetts, Amherst, 1996.

main: submitted to World Scientific on June 6, 2002 